\title{
SUB-LINEAR ESTIMATE OF LARGE VELOCITIES IN A COLLISIONLESS PLASMA*
}

\author{
ZILI $\mathrm{CHEN}^{\dagger}$ AND XIANWEN ZHANG ${ }^{\ddagger}$
}

\begin{abstract}
We consider the Vlasov-Poisson system in three space dimensions in the electrostatic case. For a smooth solution with compactly supported initial datum, the growth estimate of its velocity support is improved to $t^{\frac{2}{11}}+\varepsilon$ for any $\varepsilon>0$. As a consequence, we obtain a better decay estimate of the electrical field, namely $\|E\|_{\infty}=O\left(t^{-\frac{1}{11}+\varepsilon}\right)$ as $t \rightarrow \infty$.
\end{abstract}

Key words. Collisionless plasma, Vlasov-Poisson system, asymptotic behavior.

AMS subject classifications. 35F20, 35J05, 82D10.

\section{Introduction}

In this paper, we consider the asymptotic behavior of classical solutions to the three-dimensional Vlasov-Poisson system:

$$
\begin{aligned}
& \partial_{t} f+v \cdot \nabla_{x} f+E \cdot \nabla_{v} f=0, \quad f(0, x, v)=f_{0}(x, v), \\
& -\triangle_{x} U(t, x)=\gamma \rho(t, x), \quad \rho(t, x)=\int_{\mathbb{R}^{3}} f(t, x, v) d v, \\
& E(t, x)=-\nabla_{x} U(t, x),
\end{aligned}
$$

where the unknown $f(t, x, v) \geq 0$ denotes microscopic density of particles at time $t \geq 0$ and position $x \in \mathbb{R}^{3}$, moving with velocity $v \in \mathbb{R}^{3}$. The self-generated field $E(t, x)$ is the Coulomb field or Newtonian field, which is described by $\gamma=1$ and $\gamma=-1$ respectively. Assuming $f_{0} \in \mathcal{C}_{c}^{1}\left(\mathbb{R}^{3} \times \mathbb{R}^{3}\right)$, we know that there exists a unique solution $f \in \mathcal{C}^{1}\left(\mathbb{R}_{+} \times\right.$ $\mathbb{R}^{3} \times \mathbb{R}^{3}$ ) to this system, and the smooth solution remains compactly supported at any finite time; see $[9,12]$. So, we define for $t \geq 0$

$$
R(t)=\sup \left\{|v|: \exists x \in \mathbb{R}^{3} \text { such that } f(t, x, v) \neq 0\right\} .
$$

Several different super-linear estimates for $R(t)$ were given in $[2,9,12]$. The first sub-linear estimate was obtained in [13]:

$$
R(t) \leq C(2+t)^{11 / 15} \ln ^{4 / 15}(2+t), \quad t \geq 0
$$

which was improved in [7] with exponent $2 / 3$, namely

$$
R(t) \leq C(2+t)^{2 / 3} \ln ^{11 / 21}(2+t), \quad t \geq 0 .
$$

Another method to get the existence of a classical solution to the Vlasov-Poisson system is based on the control of moments (see [4]). For more background and related topics, see $[1,11]$ and the references therein.

*Received: December 20, 2012; accepted (in revised form): February 26, 2013. Communicated by Francois Bouchut.

†School of Mathematics and Statistics, Huazhong University of Science and Technology, Wuhan, Hubei 430074, P.R. China (chenzili_xiu@163.com).

$\ddagger$ Corresponding author. School of Mathematics and Statistics, Huazhong University of Science and Technology, Wuhan, Hubei 430074, P.R. China (xwzhang@hust.edu.cn). 
For the case of $\gamma=1$, strong a priori estimates were obtained in $[3,8]$ :

$$
\begin{gathered}
\iint(v-x / t)^{2} f(t, x, v) d v d x \leq C t^{-1}, \quad t \geq 1, \\
\|\rho(t, \cdot)\|_{5 / 3} \leq C t^{-3 / 5}, \quad t \geq 1,
\end{gathered}
$$

where the constant $C>0$ depends on $f(1)$. So it is natural to expect a better bound in this case. G. Rein gave a direct estimate in [10]: $R(t) \leq C(1+t)^{2 / 3}$. C. Pallard improved this bound to $R(t) \leq C(1+t)^{6 / 25} \ln ^{6 / 25}(2+t)$ in $[5,6]$. The purpose of this paper is to slightly refine the last estimate. Our main theorem is the following.

ThEOREM 1.1. For any nonnegative function $f_{0} \in \mathcal{C}_{c}^{1}\left(\mathbb{R}^{3} \times \mathbb{R}^{3}\right)$ and $\varepsilon>0$, there exists a constant $C_{\varepsilon}>1$ depending on $f_{0}$ and $\varepsilon$ such that

$$
R(t) \leq C_{\varepsilon}(1+t)^{\frac{2}{11}+\varepsilon}, \quad t \geq 0 .
$$

Furthermore,

$$
\|E(t, \cdot)\|_{\infty} \leq C_{\varepsilon}(1+t)^{-\frac{1}{11}+\varepsilon}, \quad t \geq 0 .
$$

Denote the characteristic flow corresponding to the first order hyperbolic partial differential equation $(1.1)$ by $(X(s, t, x, v), V(s, t, x, v))$, then

$$
\begin{cases}\frac{d X(s, t, x, v)}{d s}=V(s, t, x, v), & X(t, t, x, v)=x, \\ \frac{d V(s, t, x, v)}{d s}=E(s, X(s, t, x, v)), & V(t, t, x, v)=v .\end{cases}
$$

By uniqueness we know

$$
\left\{\begin{array}{l}
X\left(s_{1}, s_{2}, X\left(s_{2}, t, x, v\right), V\left(s_{2}, t, x, v\right)\right)=X\left(s_{1}, t, x, v\right), \\
V\left(s_{1}, s_{2}, X\left(s_{2}, t, x, v\right), V\left(s_{2}, t, x, v\right)\right)=V\left(s_{1}, t, x, v\right),
\end{array}\right.
$$

and moreover the characteristic flow is measure preserving. For the sake of simplicity, we shall use the shorthand

$$
(X(s), V(s))=(X(s, t, x, v), V(s, t, x, v))
$$

and

$$
\left(X^{*}(s), V^{*}(s)\right)=\left(X\left(s, t, x^{*}, v^{*}\right), V\left(s, t, x^{*}, v^{*}\right)\right)
$$

throughout the paper, where $\left(x^{*}, v^{*}\right)$ means that it belongs to the support of $f(t)$.

Using the notation of $[13,6]$ we define

$$
\bar{\Delta}(t, P)=\sup \left\{\triangle \in[0, t]: \forall\left(x^{*}, v^{*}\right) \in \operatorname{supp} f(t) \Rightarrow \int_{t-\Delta}^{t}\left|E\left(s, X^{*}(s)\right)\right| d s \leq P\right\}
$$

and

$$
\theta^{*}(s)=V^{*}(s)-\frac{X^{*}(s)}{s} .
$$

The analysis centers on estimating $\int_{t-\delta}^{t}\left|E\left(s, X^{*}(s)\right)\right| d s$ for some $0<\delta<t$. On the one hand, from (1.9) we know

$$
\left|V^{*}(t)-V^{*}(t-\delta)\right| \leq \int_{t-\delta}^{t}\left|E\left(s, X^{*}(s)\right)\right| d s .
$$


On the other hand, from $\left(s \theta^{*}\right)^{\prime}(s)=s E\left(s, X^{*}(s)\right)$ we obtain

$$
\theta^{*}(t)=\frac{t-\delta}{t} \theta^{*}(t-\delta)+\frac{1}{t} \int_{t-\delta}^{t} s E\left(s, X^{*}(s)\right) d s,
$$

and then

$$
\left|\theta^{*}(t)\right| \leq\left|\theta^{*}(t-\delta)\right|+\int_{t-\delta}^{t}\left|E\left(s, X^{*}(s)\right)\right| d s
$$

So, we define

$$
I=\int_{t-\delta}^{t}\left|E\left(s, X^{*}(s)\right)\right| d s
$$

The estimates for $I$ are critical in this paper. At first, we give a result obtained in [10] (see also Proposition 1 in [6] for its proof).

Lemma 1.2. There exists a constant $C>1$ such that for any $P>0$ and $t \geq 2$,

$$
\int_{t-\delta}^{t}\left|E\left(s, X^{*}(s)\right)\right| d s \leq C\left(P^{4 / 3} t^{-1 / 3} \delta+\delta^{1 / 2} t^{-1 / 2} \ln ^{1 / 2}\left[t\left(2+P^{-1}\right)\right]\right),
$$

where $\delta \leq \min \left\{t^{1 / 2}, \bar{\Delta}(t, P / 5)\right\}$.

This lemma implies a lower bound for $\bar{\Delta}(t, P)$ (see [6]).

Lemma 1.3. There exists $c_{2} \in(0,1)$ such that for any $t \geq 5$ and $P>0$,

$$
\bar{\Delta}(t, P) \geq c_{2} \min \left\{t, P^{2} t \ln ^{-1} t, P t^{5 / 7} \ln ^{-4 / 7} t\right\} .
$$

In this paper, the letter $C$ is used to denote a positive constant which changes from line to line, $c_{1}, c_{2}$, etc. denote fixed positive constants, and $\|\cdot\|_{p}$ always denotes the norm of the space $L^{p}\left(\mathbb{R}^{3}\right)$ for $1 \leq p \leq \infty$.

\section{The estimate about $I$}

The point is to get a better estimate than (1.12) for a suitable $\delta \in(0, t)$. Note that $f_{0}$ is nonnegative, and then $f$ is nonnegative, so

$$
\begin{aligned}
I & \leq \int_{t-\delta}^{t} \int_{\mathbb{R}^{3}} \frac{\rho(s, x)}{\left|x-X^{*}(s)\right|^{2}} d x d s \\
& =\int_{t-\delta}^{t} \int_{\mathbb{R}^{3}} \int_{\mathbb{R}^{3}} \frac{f(s, x, v)}{\left|x-X^{*}(s)\right|^{2}} d x d v d s .
\end{aligned}
$$

Similar to the decomposition in [13], the integral area is divided into three sets:

$$
\begin{aligned}
G & =\left\{(s, x, v) \in(t-\delta, t) \times \mathbb{R}^{3} \times \mathbb{R}^{3}: \quad\left|v-V^{*}(s)\right| \leq P \quad \text { or }|v-x / s| \leq P\right\}, \\
B & =\left\{(s, x, v) \in(t-\delta, t) \times \mathbb{R}^{3} \times \mathbb{R}^{3}: \quad\left|x-X^{*}(s)\right| \leq r(s, x, v)\right\} \backslash G, \\
U & =(t-\delta, t) \times \mathbb{R}^{3} \times \mathbb{R}^{3} \backslash(G \cup B),
\end{aligned}
$$

with

$$
\begin{gathered}
\delta(s, x, v)=c_{2} \min \left\{\frac{1}{5} s, \frac{1}{25}\left|v-V^{*}(s)\right|^{2} s \ln ^{-1} s, \frac{1}{5}\left|v-V^{*}(s)\right| s^{5 / 7} \ln ^{-4 / 7} s,\right. \\
\left.\frac{1}{25}|v-x / s|^{2} s \ln ^{-1} s, \frac{1}{5}|v-x / s| s^{5 / 7} \ln ^{-4 / 7} s\right\},
\end{gathered}
$$




$$
\begin{aligned}
r(s, x, v) & =\frac{R}{\left|v-V^{*}(s)\right||v-x / s|^{2} \delta(s, x, v)}, \\
\delta & =c_{2} \min \left\{\frac{1}{5} t, \bar{\Delta}\left(t, \frac{1}{5}\left|v^{*}-x^{*} / t\right|\right)\right\},
\end{aligned}
$$

where $c_{2}$ is the constant stated in Lemma $1.3, P$ and $R$ are undetermined parameters, and $P$ will be chosen less than $t^{-2 / 7} \ln ^{3 / 7} t$. Following from Lemma 1.3, we have

$$
\delta(t, x, v) \leq \min \left\{\frac{1}{5} t, \bar{\Delta}\left(t, \frac{1}{5}\left|v-v^{*}\right|\right), \bar{\Delta}\left(t, \frac{1}{5}|v-x / t|\right)\right\} .
$$

\subsection{The contribution of $G$. Denote}

$$
\bar{\rho}(s, x)=\int_{\left|v-V^{*}(s)\right| \leq P \text { or }|v-x / s| \leq P} f(s, x, v) d v .
$$

Following from (1.6), we have $\|\bar{\rho}(s, \cdot)\|_{5 / 3} \leq C s^{-3 / 5}$. And using the well known inequality[6]

$$
\left\|\kappa *|\cdot|^{-2}\right\|_{\infty} \leq c_{1}\|\kappa\|_{5 / 3}^{5 / 9}\|\kappa\|_{\infty}^{4 / 9}
$$

we obtain

$$
\begin{aligned}
\iiint_{G} \frac{f(s, x, v)}{\left|x-X^{*}(s)\right|^{2}} d x d v d s & \leq \int_{t-\delta}^{t} \int \frac{\bar{\rho}(s, x)}{\left|x-X^{*}(s)\right|^{2}} d x d s \\
& \leq c_{1} \int_{t-\delta}^{t}\|\bar{\rho}(s, \cdot)\|_{5 / 3}^{5 / 9}\|\bar{\rho}(s, \cdot)\|_{\infty}^{4 / 9} d s \\
& \leq C \int_{t-\delta}^{t} s^{-1 / 3} d s P^{4 / 3}
\end{aligned}
$$

that is

$$
I_{G} \leq C t^{-1 / 3} P^{4 / 3} \delta
$$

2.2. The contribution of $B$. Following the method used in [6], a further partition of $B$ will be given:

$$
B^{+}=B \cap\left\{\left|x-X^{*}(s)\right|>2 t P / 5\right\}, \quad B^{-}=B \backslash B^{+} .
$$

By the definition of $\delta(s, x, v)$ we decompose $B=\bigcup_{k=1}^{5} B_{k}$ by

$$
B_{k}=\left\{(s, x, v) \in B: \delta(s, x, v)=5^{-\frac{3+(-1)^{k}}{2}} c_{2}\left|v-V^{*}(s)\right|^{\sigma_{1 k}}|v-x / s|^{\sigma_{2 k}} s^{-\alpha_{k}} \ln ^{-\beta_{k}} s\right\}
$$

where $\sigma_{1 k}, \sigma_{2 k}, \alpha_{k}$, and $\beta_{k}(k=1, \cdots, 5)$ are respectively defined in the table.

For $s>5$ and $(s, x, v) \in B_{k}$, we have

$$
\left|x-X^{*}(s)\right|\left|v-V^{*}(s)\right|^{1+\sigma_{1 k}}|v-x / s|^{2+\sigma_{2 k}} \leq c_{3} R s^{\alpha_{k}} \ln ^{\beta_{k}} s,
$$

where $c_{3}=\frac{25}{c_{2}}$.

In the following, we denote $B^{+} \cap B_{k}$ and $B^{-} \cap B_{k}$ by $B_{k}^{+}$and $B_{k}^{-}$respectively $(k=1, \cdots, 5)$. 


\begin{tabular}{c|cccc}
$k$ & $\sigma_{1 k}$ & $\sigma_{2 k}$ & $\alpha_{k}$ & $\beta_{k}$ \\
\hline 1 & 0 & 0 & -1 & 0 \\
2 & 2 & 0 & -1 & 1 \\
3 & 1 & 0 & $-5 / 7$ & $4 / 7$ \\
4 & 0 & 2 & -1 & 1 \\
5 & 0 & 1 & $-5 / 7$ & $4 / 7$
\end{tabular}

The contribution of $B^{-}$: Pick $(s, x, v) \in B^{-}$. Note that $\delta \leq t / 5$, so we obtain $2 t P / 5 \leq s P / 2$ and hence

$$
|v-x / s| \geq\left|v-X^{*}(s) / s\right|-s^{-1}\left|X^{*}(s)-x\right| \geq\left|v-X^{*}(s) / s\right|-P / 2 .
$$

Since $|v-x / s|>P$ we have

Similarly,

$$
\left|v-X^{*}(s) / s\right| \leq \frac{3}{2}|v-x / s| .
$$

$$
\left|v-X^{*}(s) / s\right| \geq|v-x / s|-s^{-1}\left|X^{*}(s)-x\right| \geq|v-x / s|-P / 2 \geq \frac{1}{2}|v-x / s|,
$$

and thus

$$
\frac{1}{2}|v-x / s| \leq\left|v-X^{*}(s) / s\right| \leq \frac{3}{2}|v-x / s| .
$$

Combining (2.4) and (2.5) we obtain that

$$
\left|x-X^{*}(s)\right|\left|v-V^{*}(s)\right|^{1+\sigma_{1 k}}\left|v-X^{*}(s) / s\right|^{2+\sigma_{2 k}} \leq c_{3}^{\prime} R s^{\alpha_{k}} \ln ^{\beta_{k}} s,
$$

where $c_{3}^{\prime}=\left(\frac{3}{2}\right)^{4} c_{3}$. So integrating in the $x$ variable first gives:

$$
\begin{aligned}
& I_{B_{k}^{-}}=\int_{B_{k}^{-}} \frac{f(s, x, v)}{\left|x-X^{*}(s)\right|^{2}} d x d v d s \\
& \leq c_{3}^{\prime}\left\|f_{0}\right\|_{L^{\infty}\left(\mathbb{R}^{3} \times \mathbb{R}^{3}\right)} \int_{t-\delta}^{t} R s^{\alpha_{k}} \ln ^{\beta_{k}} s \\
& \cdot\left(\int_{\left|v-V^{*}(s)\right|>P \text { and }\left|v-X^{*}(s) / s\right|>P / 2} \frac{1}{\left|v-V^{*}(s)\right|^{1+\sigma_{1 k}}\left|v-X^{*}(s) / s\right|^{2+\sigma_{2 k}}} d v\right) d s \\
& \leq C \int_{t-\delta}^{t} R s^{\alpha_{k}} \ln ^{\beta_{k}} s \\
& \cdot\left(\int_{\left|v-V^{*}(s)\right|>P \text { and }\left|v-X^{*}(s) / s\right|>P / 2} \frac{1}{\left|v-V^{*}(s)\right|^{1+\sigma_{1 k}}\left|v-X^{*}(s) / s\right|^{2+\sigma_{2 k}}} d v\right) d s \text {. }
\end{aligned}
$$

Firstly, note that by the definition of $R(t)$ we have that $|v|,\left|V^{*}(s)\right| \leq R(s)$. Then assuming that there exists positive constants $\gamma$ and $C$ such that $P>C t^{-\gamma}$ for any $t>1$, we can directly compute that

$$
\begin{aligned}
I_{B_{1}^{-}} \leq C R & \int_{t-\delta}^{t} s^{-1} d s \int_{P / 2<\left|v-X^{*}(s) / s\right| \leq\left|v-V^{*}(s)\right| \leq 2 R(s)} \frac{1}{\left|v-V^{*}(s)\right|\left|v-X^{*}(s) / s\right|^{2}} d v \\
& +C R \int_{t-\delta}^{t} s^{-1} d s \int_{P<\left|v-V^{*}(s)\right| \leq\left|v-X^{*}(s) / s\right|} \frac{1}{\left|v-V^{*}(s)\right|\left|v-X^{*}(s) / s\right|^{2}} d v
\end{aligned}
$$




$$
\begin{aligned}
& \leq C R \int_{t-\delta}^{t} s^{-1} d s \int_{P / 2<\left|v-X^{*}(s) / s\right| \leq 2 R(s)} \frac{1}{\left|v-X^{*}(s) / s\right|^{3}} d v \\
& \quad+C R \int_{t-\delta}^{t} s^{-1} d s \int_{P<\left|v-V^{*}(s)\right| \leq 2 R(s)} \frac{1}{\left|v-V^{*}(s)\right|^{3}} d v \\
& \leq C R \int_{t-\delta}^{t} s^{-1} \ln s d s .
\end{aligned}
$$

Now, we consider $B_{2}^{-}$. The definition of $\delta(s, x, v)$ implies that

$$
\left|v-V^{*}(s)\right| \leq|v-x / s|
$$

Combining this with (2.5), we obtain $\left|v-V^{*}(s)\right| \leq 2\left|v-X^{*}(s) / s\right|$. Following from

$$
\left|v-V^{*}(s)\right|+\left|v-X^{*}(s) / s\right| \geq\left|V^{*}(s)-X^{*}(s) / s\right|=\left|\theta^{*}(s)\right|
$$

we have

$$
\left|v-X^{*}(s) / s\right| \geq \frac{1}{3}\left|\theta^{*}(s)\right|
$$

Consequently,

$$
\begin{aligned}
I_{B_{2}^{-}} \leq & C \int_{t-\delta}^{t} R s^{-1} \ln s \\
& \cdot\left(\int_{\left|v-X^{*}(s) / s\right| \geq \frac{1}{3}\left|\theta^{*}(s)\right| \text { and } P<\left|v-V^{*}(s)\right| \leq 2 R(s)} \frac{1}{\left|v-V^{*}(s)\right|^{3}\left|v-X^{*}(s) / s\right|^{2}} d v\right) d s \\
\leq & C \int_{t-\delta}^{t} R s^{-1} \ln s d s \int_{P<\left|v-V^{*}(s)\right| \leq 2 R(s)} \frac{1}{\left|v-V^{*}(s)\right|^{3}\left|\theta^{*}(s)\right|^{2}} d v \\
\leq & C R \int_{t-\delta}^{t} \frac{s^{-1} \ln ^{2} s}{\left|\theta^{*}(s)\right|^{2}} d s .
\end{aligned}
$$

Similarly, for $B_{3}^{-}$we also have $\left|v-X^{*}(s) / s\right| \geq \frac{1}{3}\left|\theta^{*}(s)\right|$ and $\left|v-V^{*}(s)\right| \leq 2 \mid v-$ $X^{*}(s) / s \mid$. Then we have $B_{3}^{-}=\left(B_{3}^{1} \cup B_{3}^{2}\right)$, where

and

$$
B_{3}^{1}=\left\{(s, x, v) \in B_{3}^{-}: \quad \frac{2}{3}\left|\theta^{*}(s)\right| \leq\left|v-V^{*}(s)\right| \leq 2\left|v-X^{*}(s) / s\right|\right\}
$$

$$
B_{3}^{2}=\left\{(s, x, v) \in B_{3}^{-}: \quad\left|v-X^{*}(s) / s\right| \geq \frac{1}{3}\left|\theta^{*}(s)\right| \geq \frac{1}{2}\left|v-V^{*}(s)\right|\right\} .
$$

For the first one,

$$
\begin{aligned}
I_{B_{3}^{1}} \leq & C \int_{t-\delta}^{t} R s^{-5 / 7} \ln ^{4 / 7} s \\
& \cdot\left(\int_{\frac{2}{3}\left|\theta^{*}(s)\right| \leq\left|v-V^{*}(s)\right| \leq 2\left|v-X^{*}(s) / s\right|} \frac{1}{\left|v-V^{*}(s)\right|^{2}\left|v-X^{*}(s) / s\right|^{2}} d v\right) d s \\
\leq & C \int_{t-\delta}^{t} R s^{-5 / 7} \ln ^{4 / 7} s d s \int_{\left|v-V^{*}(s)\right| \geq \frac{2}{3}\left|\theta^{*}(s)\right|} \frac{1}{\left|v-V^{*}(s)\right|^{4}} d v \\
\leq & C R \int_{t-\delta}^{t} \frac{s^{-5 / 7} \ln ^{4 / 7} s}{\left|\theta^{*}(s)\right|} d s .
\end{aligned}
$$


For the second one,

$$
\begin{aligned}
I_{B_{3}^{2} \leq} \leq & C \int_{t-\delta}^{t} R s^{-5 / 7} \ln ^{4 / 7} s \\
& \cdot\left(\int_{\left|v-X^{*}(s) / s\right| \geq \frac{1}{3}\left|\theta^{*}(s)\right| \text { and }\left|v-V^{*}(s)\right| \leq \frac{2}{3}\left|\theta^{*}(s)\right|} \frac{1}{\left|v-V^{*}(s)\right|^{2}\left|v-X^{*}(s) / s\right|^{2}} d v\right) d s \\
\leq & C \int_{t-\delta}^{t} R s^{-5 / 7} \ln ^{4 / 7} s d s \int_{\left|v-V^{*}(s)\right| \leq \frac{2}{3}\left|\theta^{*}(s)\right|} \frac{1}{\left|v-V^{*}(s)\right|^{2}\left|\theta^{*}(s)\right|^{2}} d v \\
\leq & C R \int_{t-\delta}^{t} \frac{s^{-5 / 7} \ln ^{4 / 7} s}{\left|\theta^{*}(s)\right|} d s .
\end{aligned}
$$

So,

$$
I_{B_{3}^{-}}=I_{B_{3}^{1}}+I_{B_{3}^{2}} \leq C R \int_{t-\delta}^{t} \frac{s^{-5 / 7} \ln ^{4 / 7} s}{\left|\theta^{*}(s)\right|} d s
$$

For $I_{B_{4}^{-}}$, the definition of $\delta(s, x, v)$ implies that

$$
\left|v-V^{*}(s)\right| \geq|v-x / s| .
$$

So $\left|v-V^{*}(s)\right| \geq \frac{2}{3}\left|v-X^{*}(s) / s\right|$ because of $(2.5)$, and then $\left|v-X^{*}(s) / s\right| \leq 3 R(s)$. By the same computation as $B_{2}^{-}$, we obtain

$$
\left|v-V^{*}(s)\right| \geq \frac{3}{5}\left|\theta^{*}(s)\right|
$$

Then, following the method used in $[6,13]$ we obtain

$$
\begin{aligned}
I_{B_{4}^{-}} \leq & C \int_{t-\delta}^{t} R s^{-1} \ln s \\
& \cdot\left(\int_{\left|v-V^{*}(s)\right| \geq \frac{3}{5}\left|\theta^{*}(s)\right| \text { and } P / 2<\left|v-X^{*}(s) / s\right| \leq 3 R(s)} \frac{1}{\left|v-V^{*}(s)\right|\left|v-X^{*}(s) / s\right|^{4}} d v\right) d s \\
\leq & C \int_{t-\delta}^{t} R s^{-1} \ln s d s \int_{P / 2<\left|v-X^{*}(s) / s\right| \leq 3 R(s)} \frac{1}{P\left|v-X^{*}(s) / s\right|^{3}\left|\theta^{*}(s)\right|} d v \\
\leq & C R P^{-1} \int_{t-\delta}^{t} \frac{s^{-1} \ln ^{2} s}{\left|\theta^{*}(s)\right|} d s .
\end{aligned}
$$

Analogously, we obtain

$$
I_{B_{5}^{-}}=C R \int_{t-\delta}^{t} \frac{s^{-5 / 7} \ln ^{11 / 7} s}{\left|\theta^{*}(s)\right|} d s
$$

Thus

$$
\begin{aligned}
I_{B^{-}} \leq C R\left\{\int_{t-\delta}^{t} s^{-1} \ln s d s\right. & +\int_{t-\delta}^{t} \frac{s^{-1} \ln s}{\left|\theta^{*}(s)\right|^{2}} d s \\
& \left.+\int_{t-\delta}^{t} \frac{s^{-5 / 7} \ln ^{11 / 7} s}{\left|\theta^{*}(s)\right|} d s+P^{-1} \int_{t-\delta}^{t} \frac{s^{-1} \ln ^{2} s}{\left|\theta^{*}(s)\right|} d s\right\} .
\end{aligned}
$$


Remember that $\delta=c_{2} \min \left\{\frac{1}{5} t, \bar{\Delta}\left(t, \frac{1}{5}\left|v^{*}-x^{*} / t\right|\right)\right\}$, so by the same arguments used in Lemma 2.2 below, we have

$$
\frac{3}{5}\left|v^{*}-x^{*} / t\right| \leq\left|V^{*}(s)-\frac{X^{*}(s)}{s}\right| \leq \frac{7}{5}\left|v^{*}-x^{*} / t\right| .
$$

Thus,

$$
\begin{aligned}
& I_{B^{-}} \leq C R\left[\delta t^{-1} \ln t+\delta t^{-1} \ln t\left|\theta^{*}(t)\right|^{-2}\right. \\
&\left.+\delta t^{-5 / 7} \ln ^{11 / 7} t\left|\theta^{*}(t)\right|^{-1}+\delta P^{-1} t^{-1} \ln ^{2} t\left|\theta^{*}(t)\right|^{-1}\right] \\
& \leq C R\left[\delta t^{-1} \ln t+\delta t^{-1} \ln t\left|\theta^{*}(t)\right|^{-2}+\delta P^{-1} t^{-1} \ln ^{2} t\left|\theta^{*}(t)\right|^{-1}\right]
\end{aligned}
$$

since $P \leq t^{-2 / 7} \ln ^{3 / 7} t$.

The contribution of $B^{+}$: For $B_{k}^{+}, k=2,3,4,5$, we know $\left|v-V^{*}(s)\right| \leq|v-x / s|$ or $\left|v-V^{*}(s)\right| \geq|v-x / s|$ because of the definition of $\delta(s, x, v)$. Then,

$$
\left|x-X^{*}(s)\right||v-\star|^{3+\sigma_{1 k}+\sigma_{2 k}} \leq c_{2} R s^{\alpha_{k}} \ln ^{\beta_{k}} s,
$$

where $\star=V^{*}(s)$ or $x / s$. Writing $x=X^{*}(s)+\rho \omega$ with $\rho \geq \frac{2 t P}{5}$ and $\omega \in \mathbb{S}^{2}$, we find

$$
\rho|v-\star|^{3-\epsilon_{k}} P^{\epsilon_{k}+\sigma_{1 k}+\sigma_{2 k}} \leq c_{2} R s^{\alpha_{k}} \ln ^{\beta_{k}} s, \quad \epsilon_{k} \in(0,3),
$$

since $(s, x, v) \in B_{k}^{+}$and $B_{k}^{+} \cap G=\emptyset$. Then

$$
\begin{aligned}
I_{B_{k}^{+}} & \leq C \int_{t-\delta}^{t} \int_{\frac{2 t P}{5}}^{\infty} \int_{\mathbb{S}^{2}} \int_{\mathbb{R}^{3}} 1_{B_{k}^{+}}\left(s, X^{*}(s)+\rho \omega, v\right) d v d \omega d \rho d s \\
& \leq C R^{\frac{3}{3-\epsilon_{k}}} P^{-\frac{3\left(\epsilon_{k}+\sigma_{1 k}+\sigma_{2 k}\right)}{3-\epsilon_{k}}} \int_{\frac{2 t P}{5}}^{\infty} \rho^{-\frac{3}{3-\epsilon_{k}}} d \rho \int_{t-\delta}^{t} s^{\frac{3 \alpha_{k}}{3-\epsilon_{k}}} \ln ^{\frac{3 \beta_{k}}{3-\epsilon_{k}}} s d s \\
& \leq C \delta R^{\frac{3}{3-\epsilon_{k}}} P^{-\frac{3\left(\epsilon_{k}+\sigma_{1 k}+\sigma_{2 k}\right)+\epsilon_{k}}{3-\epsilon_{k}}} t^{\frac{3 \alpha_{k}-\epsilon_{k}}{3-\epsilon_{k}}} \ln ^{\frac{3 \beta_{k}}{3-\epsilon_{k}}} t
\end{aligned}
$$

Actually, we can choose $\epsilon_{2}=\epsilon_{4}=\epsilon$ and $\epsilon_{3}=\epsilon_{5}=\epsilon^{\prime}$ to obtain

$$
\begin{aligned}
& I_{B_{2}^{+}}=I_{B_{4}^{+}} \leq C \delta R^{\frac{3}{3-\epsilon}} P^{-\frac{4 \epsilon+6}{3-\epsilon}} t^{\frac{-3-\epsilon}{3-\epsilon}} \ln ^{\frac{3}{3-\epsilon}} t, \\
& I_{B_{3}^{+}}=I_{B_{5}^{+}} \leq C \delta R^{\frac{3}{3-\epsilon^{\prime}}} P^{-\frac{4 \epsilon^{\prime}+3}{3-\epsilon^{\prime}}} t^{\frac{-15 / 7-\epsilon^{\prime}}{3-\epsilon^{\prime}}} \ln ^{\frac{12 / 7}{3-\epsilon^{\prime}}} t .
\end{aligned}
$$

For $I_{B_{1}^{+}}$, by dividing the integral area into two parts $-\left|v-V^{*}(s)\right| \leq|v-x / s|$ and $\mid v-$ $V^{*}(s)|>| v-x / s \mid$-we can similarly compute that

$$
I_{B_{1}^{+}} \leq C \delta R^{\frac{3}{3-\epsilon_{1}}} P^{-\frac{4 \epsilon_{1}}{3-\epsilon_{1}}} t^{\frac{-3-\epsilon_{1}}{3-\epsilon_{1}}}
$$

If $P \leq t^{-2 / 7} \ln ^{3 / 7} t$, then for any $\epsilon \in(0,3)$ we can choose $\epsilon_{1}=\epsilon^{\prime}=\epsilon$ to get $I_{B_{3}^{+}} \leq I_{B_{2}^{+}}$ and $I_{B_{1}^{+}} \leq C I_{B_{2}^{+}}$. As a consequence, we have

$$
I_{B^{+}} \leq C \delta R^{\frac{3}{3-\epsilon}} P^{-\frac{4 \epsilon+6}{3-\epsilon}} t^{\frac{-3-\epsilon}{3-\epsilon}} \ln ^{\frac{3}{3-\epsilon}} t
$$

for $P \leq t^{-2 / 7} \ln ^{3 / 7} t$. Combining (2.6) and (2.7), we obtain

$$
\begin{aligned}
& \frac{1}{\delta} I_{B} \leq C\left[R t^{-1} \ln t+R^{\frac{3}{3-\epsilon}} P^{-\frac{4 \epsilon+6}{3-\epsilon}} t^{\frac{-3-\epsilon}{3-\epsilon}} \ln ^{\frac{3}{3-\epsilon}} t\right. \\
& \left.+R t^{-1} \ln t\left|\theta^{*}(t)\right|^{-2}+R P^{-1} t^{-1} \ln ^{2} t\left|\theta^{*}(t)\right|^{-1}\right]
\end{aligned}
$$

for $P \leq t^{-2 / 7} \ln ^{3 / 7} t$. 
2.3. The contribution of $U$. Note that the characteristic flow is measure preserving and $f(s, X(s), V(s))=f(t, x, v)$, so we have

$$
\iiint_{U} \frac{f(s, x, v)}{\left|x-X^{*}(s)\right|^{2}} d x d v d s=\iint\left(\int_{t-\delta}^{t} \frac{1_{U}(s, X(s), V(s))}{\left|X(s)-X^{*}(s)\right|^{2}} d s\right) f(t, x, v) d v d x .
$$

It is natural to follow the classic method used in [9, 12], and especially in $[13,6]$, to control the inner time-integral. The next lemma is the main tool.

Lemma 2.1. Let $s \mapsto(X(s), V(s))$ be a characteristic curve and $\Delta t \in[0, t]$ satisfying

$$
\Delta t \leq \bar{\Delta}\left(t, \frac{1}{5}\left|v-v^{*}\right|\right) .
$$

Suppose there exists some $D>0$ such that for any $s \in(t-\Delta t, t)$

$$
r(s, X(s), V(s)) \geq \operatorname{Dr}(t, x, v),
$$

whenever $(s, X(s), V(s)) \in U$. Then the following upper bound holds true:

$$
\int_{t-\Delta t}^{t} \frac{1_{U}(s, X(s), V(s))}{\left|X(s)-X^{*}(s)\right|^{2}} d s \leq \frac{1}{D\left|v-v^{*}\right| r(t, x, v)} .
$$

This lemma means that by controlling $\Delta t$ we could bound $I$ in the ugly set. For its proof we refer the readers to [13].

Now we show that the conditions of Lemma 2.1 are satisfied for $\Delta t=\delta(t, x, v)$. Remember (2.1), so we only need to prove the following result.

Lemma 2.2. There exists a constant $c_{4}>0$ such that

$$
r(s, X(s), V(s)) \geq c_{4} r(t, x, v),
$$

for any $t \geq 5$ and $s \in(t-\delta(t, x, v), t)$.

Proof. Following from (2.1), we have

$$
\delta(t, x, v) \leq \bar{\Delta}\left(t, \frac{1}{5}\left|v-v^{*}\right|\right),
$$

which yield that for any $s \in(t-\delta(t, x, v), t)$,

$$
|V(s)-v| \leq \frac{1}{5}\left|v-v^{*}\right|
$$

and

$$
\left|V^{*}(s)-v^{*}\right| \leq \frac{1}{5}\left|v-v^{*}\right| .
$$

As a consequence, we obtain

$$
\frac{3}{5}\left|v-v^{*}\right| \leq\left|V(s)-V^{*}(s)\right| \leq \frac{7}{5}\left|v-v^{*}\right| .
$$

Now considering

$$
\theta(s)=V(s)-\frac{X(s)}{s} .
$$


Similar to (1.10), we obtain for any $s \in(t-\delta(t, x, v), t)$

$$
|\theta(s)-\theta(t)| \leq \delta(t, x, v) t^{-1}|\theta(t)|+\int_{s}^{t}|E(\tau, X(\tau))| d \tau .
$$

Using (2.1) again we have $\delta(t, x, v) \leq \bar{\Delta}\left(t, \frac{1}{5}|v-x / t|\right)$ and

$$
\delta(t, x, v) \leq \frac{1}{5} t
$$

Hence

$$
\frac{3}{5}|v-x / t| \leq\left|V(s)-\frac{X(s)}{s}\right| \leq \frac{7}{5}|v-x / t| .
$$

Combining (2.10), (2.11), (2.12), and the definition of $r$, we get the desired result. $\square$

Then, using Lemma 2.1 we obtain, for any $t \geq 5$,

$$
\begin{aligned}
\int_{t-\delta(t, x, v)}^{t} \frac{1_{U}(s, X(s), V(s))}{\left|X(s)-X^{*}(s)\right|^{2}} d s & \leq \frac{1}{c_{4}\left|v-v^{*}\right| r(t, x, v)}=\frac{\delta(t, x, v)|v-x / t|^{2}}{c_{4} R} \\
& \leq c_{5} R^{-1} \int_{t-\delta(t, x, v)}^{t}\left|V(s)-\frac{X(s)}{s}\right|^{2} d s .
\end{aligned}
$$

The last inequality follows from (2.12), where $c_{5}=\frac{5}{3 c_{4}}$.

Now we will use a lemma which is introduced in [13] to make an inequality like (2.13) satisfied for some $\delta \geq \delta(t, x, v)$. It will be used in the similar way in our paper.

Lemma 2.3. Given $T>0$, suppose $a, b \in L^{1}\left((0, T) ; \mathbb{R}^{+}\right)$and $d \in \mathcal{C}\left((0, T) ; \mathbb{R}^{+}\right)$satisfy, for any $t \in(0, T)$, the inequalities $d(t) \leq t$ and

$$
\int_{t-d(t)}^{t} a(s) d s \leq \int_{t-d(t)}^{t} b(s) d s .
$$

Assume further that $a(s)=0$ whenever $d(s)=0$. Then for any $t \in(0, T)$ and $\delta \in[d(t), t]$ we have

$$
\int_{t-\delta}^{t} a(s) d s \leq 2 \int_{t-\delta}^{t} b(s) d s
$$

Define functions $a$ and $b$ as

$$
a(s)=\frac{1_{U}(s, X(s), V(s))}{\left|X(s)-X^{*}(s)\right|^{2}} 1_{s \geq 5}, \quad b(s)=\frac{c_{5}}{R}\left|V(s)-\frac{X(s)}{s}\right|^{2} 1_{s \geq 4} .
$$

Set $d(s)=\delta(s, X(s), V(s)), \quad s \in[5, \infty)$, and $d(s)=d(5) s / 5, \quad s \in(0,5)$. Note that $\delta(t, x, v) \leq t / 5$ and we have $t-\delta(t, x, v) \geq 4$ for any $t \geq 5$, which yields that

$$
\int_{t-d(t)}^{t} a(s) d s \leq \int_{t-d(t)}^{t} b(s) d s, \quad \forall t>0 .
$$

Furthermore, for $s \geq 5, d(s)=0$ implies either $V(s)=V^{*}(s)$ or $V(s)=\frac{X(s)}{s}$, so $(s, X(s), V(s)) \notin U$. Note that $(x, v)$ always belongs to the compact support of $f(t)$ when considering $I_{U}$. So, if

$$
\left|v^{*}-x^{*} / t\right| \geq|v-x / t|, \quad \forall(x, v) \in \operatorname{supp} f(t),
$$


we have $\bar{\Delta}\left(t, \frac{1}{5}\left|v^{*}-x^{*} / t\right|\right) \geq \bar{\Delta}\left(t, \frac{1}{5}|v-x / t|\right)$, and then $\delta \geq d(t)$ because of (2.1). Thus, by Lemma 2.3 we have

$$
\int_{t-\delta}^{t} a(s) d s \leq 2 \int_{t-\delta}^{t} b(s) d s, \quad \forall t>0 .
$$

Observe that $\delta \leq t / 5$, we could obtain

$$
\int_{t-\delta}^{t} \frac{1_{U}(s, X(s), V(s))}{\left|X(s)-X^{*}(s)\right|^{2}} d s \leq 2 c_{5} R^{-1} \int_{t-\delta}^{t}\left|V(s)-\frac{X(s)}{s}\right|^{2} d s, \quad \forall t \geq 7,
$$

which yields

$$
\begin{aligned}
\int_{U} \frac{f(t, x, v)}{\left|X(s)-X^{*}(s)\right|^{2}} d x d v d s & =\iiint_{t-\delta}^{t} \frac{1_{U}(s, X(s), V(s))}{\left|X(s)-X^{*}(s)\right|^{2}} d s f(t, x, v) d v d x \\
& \leq 2 c_{5} R^{-1} \int_{t-\delta}^{t} \iint\left|V(s)-\frac{X(s)}{s}\right|^{2} f(t, x, v) d v d x d s \\
& =2 c_{5} R^{-1} \int_{t-\delta}^{t} \iint\left|v-\frac{x}{s}\right|^{2} f(s, x, v) d v d x d s,
\end{aligned}
$$

for any $t \geq 7$. Considering the estimate (1.5) we obtain

$$
I_{U} \leq C R^{-1} t^{-1} \delta
$$

At last, we combine the estimates of $I_{G}, I_{B}$, and $I_{U}$ and choose proper $P, R$ to get the growth of $I$.

Proposition 2.4. If $\delta=c_{2} \min \left\{\frac{1}{5} t, \bar{\Delta}\left(t, \frac{1}{5}\left|v^{*}-x^{*} / t\right|\right)\right\},\left|\theta^{*}(t)\right|=\left|v^{*}-x^{*} / t\right| \geq|v-x / t|$ for any $(x, v) \in \operatorname{supp} f(t)$ and $\left|\theta^{*}(t)\right|>1$, then there exists a positive constant $C_{\varepsilon}$ depending on $f_{0}$ and $\varepsilon$ such that for any $t \geq 7$

$$
I=\int_{t-\delta}^{t}\left|E\left(s, X^{*}(s)\right)\right| d s \leq C_{\varepsilon} \delta t^{\frac{2}{11}+\varepsilon-1} .
$$

Proof. Following from (2.3), (2.8), and (2.16), we have for $P \leq t^{-2 / 7} \ln ^{3 / 7} t$,

$$
\begin{gathered}
\frac{1}{\delta} I \leq C\left[P^{4 / 3} t^{-1 / 3}+R^{-1} t^{-1}+R t^{-1} \ln t+R^{\frac{3}{3-\epsilon}} P^{-\frac{4 \epsilon+6}{3-\epsilon}} t^{\frac{-3-\epsilon}{3-\epsilon}} \ln { }^{\frac{3}{3-\epsilon}} t\right. \\
\left.+R t^{-1} \ln t\left|\theta^{*}(t)\right|^{-2}+R P^{-1} t^{-1} \ln ^{2} t\left|\theta^{*}(t)\right|^{-1}\right] .
\end{gathered}
$$

Let

$$
P^{4 / 3} t^{-1 / 3}=R^{-1} t^{-1}=R^{\frac{3}{3-\epsilon}} P^{-\frac{4 \epsilon+6}{3-\epsilon}} t^{\frac{-3-\epsilon}{3-\epsilon}} \ln ^{\frac{3}{3-\epsilon}} t
$$

then we obtain

$$
P=t^{-\frac{12+4 \epsilon}{42+8 \epsilon}} \ln \frac{9}{42+8 \epsilon} t, \quad R=t^{-\frac{6}{21+4 \epsilon}} \ln ^{-\frac{21}{21+4 \epsilon}} t .
$$

Taking $\epsilon=3^{-}$, we have that $R t^{-1} \ln t, R t^{-1} \ln t\left|\theta^{*}(t)\right|^{-2}$, and $R P^{-1} t^{-1} \ln ^{2} t\left|\theta^{*}(t)\right|^{-1}$ are less than

$$
C_{\epsilon} R^{-1} t^{-1}=C_{\epsilon} t^{-1+\frac{6}{21+4 \epsilon}} \ln ^{\frac{21}{21+4 \epsilon}} t .
$$

Consequently, for any $t \geq 7$ and $\varepsilon>0$, there exists a constant $C_{\varepsilon}>0$ such that

$$
\frac{1}{\delta} I \leq C_{\varepsilon} t^{\frac{2}{11}+\varepsilon-1}
$$

This completes the proof. 


\section{The proof of Theorem 1.1}

Using Proposition 2.4 we can obtain a uniform estimate on the size of $\theta^{*}(t)$.

Lemma 3.1. For any $t \geq 1$, there exists a constant $C_{\varepsilon}>1$ depending on $f_{0}$ and $\varepsilon$ such that

$$
\left|\theta^{*}(t)\right| \leq C_{\varepsilon}(t+1)^{\frac{2}{11}+\varepsilon}
$$

Proof. We only need to show that

$$
\sup _{\left(x^{*}, v^{*}\right) \in \operatorname{supp} f(t)}\left|\theta^{*}(t)\right| \leq C_{\varepsilon}(t+1)^{\frac{2}{11}+\varepsilon} .
$$

For the sake of simplicity, we still record it as $\left|\theta^{*}(t)\right|$. For any fixed $t>7$, if $\left|\theta^{*}(t)\right| \leq 1$, then (3.1) is proved. Now suppose $\left|\theta^{*}(t)\right|>1$. Following from Proposition 2.4 and inequality (1.11), we obtain that

$$
\left|\theta^{*}(t)\right| \leq\left|\theta^{*}(t-\delta)\right|+C_{\varepsilon} t^{\frac{2}{11}+\varepsilon-1} \delta
$$

for $\delta=c_{2} \min \left\{\frac{1}{5} t, \bar{\Delta}\left(t, \frac{1}{5}\left|v^{*}-x^{*} / t\right|\right)\right\}$. Let $t_{0}=t, t_{n+1}=t_{n}-\delta_{n}$ where

$$
\delta_{n}=c_{2} \min \left\{\frac{1}{5} t_{n}, \bar{\Delta}\left(t_{n}, \frac{1}{5}\left|\theta^{*}\left(t_{n}\right)\right|\right)\right\} .
$$

Continue until either $t_{n} \leq 7$ or $\left|\theta^{*}\left(t_{n}\right)\right| \leq 1$. By Lemma 1.3, we know that $t_{n}>7$ and $\left|\theta^{*}\left(t_{n}\right)\right|>1$ imply $\bar{\Delta}\left(t_{n}, \frac{1}{5}\left|\theta^{*}\left(t_{n}\right)\right|\right) \geq C$, and hence $\delta_{n} \geq C$. Therefore there exists a positive integer $N$ such that

$$
\left\{\begin{array}{l}
t_{n}>7 \text { and } \theta^{*}\left(t_{n}\right)>1, \quad 0 \leq n<N \\
t_{N} \leq 7 \text { or } \theta^{*}\left(t_{N}\right) \leq 1
\end{array}\right.
$$

By $(3.2)$,

$$
\left|\theta^{*}\left(t_{n}\right)\right| \leq\left|\theta^{*}\left(t_{n+1}\right)\right|+C_{\varepsilon} t_{n}^{\frac{2}{11}+\varepsilon-1} \delta_{n}, \quad 0 \leq n<N .
$$

Then, for any $t>7$

$$
\begin{aligned}
\left|\theta^{*}(t)\right| & \leq\left|\theta^{*}\left(t_{N}\right)\right|+C_{\varepsilon} \sum_{n=0}^{N} \delta_{n} t_{n}^{\frac{2}{11}+\varepsilon-1} \\
& \leq\left|\theta^{*}\left(t_{N}\right)\right|+C_{\varepsilon}\left(t-t_{N}\right) t^{\frac{2}{11}+\varepsilon-1} \\
& \leq C_{\varepsilon}(1+t)^{\frac{2}{11}+\varepsilon}
\end{aligned}
$$

since $\theta^{*}\left(t_{N}\right)$ is less than 1 or bounded by a positive constant which only depends on $f_{0}$. As a consequence, there exists a constant $C_{\varepsilon}>1$ depending on $f_{0}$ and $\varepsilon$ such that

$$
\left|\theta^{*}(t)\right| \leq C_{\varepsilon}(1+t)^{\frac{2}{11}+\varepsilon}
$$

for any $t \geq 1$.

Proof of Theorem 1.1.

Proof. Note that

$$
\frac{d}{d t}\left(\frac{X^{*}(s)}{s}\right)=\frac{\theta^{*}(s)}{s}, \quad s>0 .
$$


Then using Lemma 3.1, we obtain

$$
\frac{\left|x^{*}\right|}{t} \leq C_{\varepsilon}(1+t)^{\frac{2}{11}+\varepsilon}, \quad t \geq 1
$$

Combining it with (3.1), we get

$$
\begin{aligned}
\left|v^{*}\right| & \leq\left|\theta^{*}(t)\right|+\frac{\left|x^{*}\right|}{t} \\
& \leq C_{\varepsilon}(1+t)^{\frac{2}{11}+\varepsilon}
\end{aligned}
$$

for $t \geq 1$, and hence for $t \geq 0$. Using the inequality (2.2) again, we obtain (1.8) because of (1.6) and (1.7).

Acknowledgment. The authors would like to thank the anonymous referee for helpful comments and valuable suggestions concerning the presentation of this paper. This research is supported by "The Fundamental Research Funds for the Central Universities" (Project No. 2012TS008).

\section{REFERENCES}

[1] R. Glassey, The Cauchy Problem in Kinetic Theory, SIAM, Philadelphia, 1996.

[2] E. Horst, On the asymptotic growth of the solutions of the Vlasov-Poisson system, Math. Meth. Appl. Sci., 16, 75-85, 1993.

[3] R. Illner and G. Rein, Time decay of the solutions of the Vlasov-Poisson system in the plasma physical case, Math. Meth. Appl. Sci., 19, 1409-1413, 1996.

[4] P.L. Lions and B. Perthame, Propagation of moments and regularity for the 3-dimensional Vlasov-Poisson system, Inv. Math., 105, 415-430, 1991.

[5] C. Pallard, A note on the growth of velocities in a collisionless plasma, Math. Meth. Appl. Sci., 34, 803-806, 2011.

[6] C. Pallard, Growth estimates and uniform decay for a collisionless plasma, Kin. Rel. Models, 4, 549-567, 2011.

[7] C. Pallard, Large velocities in a collisionless plasma, J. Diff. Equ., 252, 2864-2876, 2012.

[8] B. Perthame, Time decay, propagation of low moments and dispersive effects for kinetic equations, Comm. Part. Diff. Equ., 21, 659-686, 1996.

[9] K. Pfaffelmoser, Global classical solutions of the Vlasov-Poisson system in three dimensions for general initial data, J. Diff. Equ., 95, 281-303, 1992.

[10] G. Rein, Growth estimates for the solutions of the Vlasov-Poisson system in the plasma physics case, Math. Nachr., 191, 269-278, 1998.

[11] G. Rein, Collisionless kinetic rquations from astrophysics-The Vlasov-Poisson system, in Handbook of Differential Equations: Evolutionary Equations, C.M. Dafermos and E. Feireisl (eds.), 3(5), 383-476, Elsevier, 2007.

[12] J. Schaeffer, Global existence of smooth solutions to the Vlasov-Poisson system in three dimensions, Comm. Part. Diff. Equ., 16, 1313-1335, 1991.

[13] J. Schaeffer, Asymptotic growth bounds for the Vlasov-Poisson system, Math. Meth. Appl. Sci., $34,262-277,2011$. 